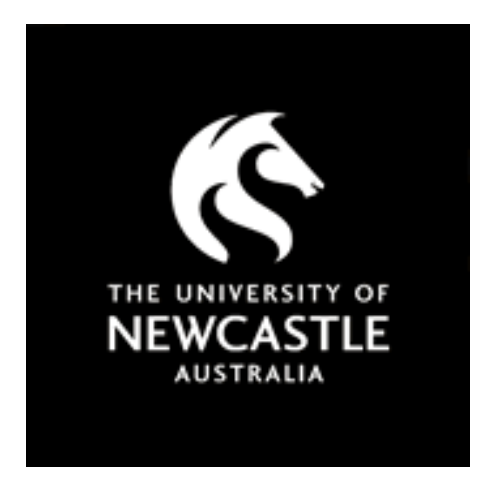

NOVA

University of Newcastle Research Online

nova.newcastle.edu.au

Morse, Andrew J.; Giakoumatos, Emma C.; Tan, Sin-Ying; Webber, Grant B.; Armes, Steven P.; Ata, Seher; Wanless, Erica J. "Giant pH-responsive microgel colloidosomes: preparation, interaction dynamics and stability", Published in Soft Matter Vol. 12, Issue 5, p. 1477-1486. (2016)

Available from: $\underline{\text { http://dx.doi.org/10.1039/c5sm02450a }}$

Accessed from: http://hdl.handle.net/1959.13/1321238 


\title{
Giant pH-Responsive Microgel Colloidosomes: Preparation, Interaction Dynamics and Stability
}

Received 00th January 20xx, Accepted 00th January 20xx DOI: $10.1039 / \times 0 \times x 00000 x$ www.rsc.org/

\author{
Andrew J. Morse, ${ }^{a}$ Emma C. Giakoumatos, ${ }^{b}$ Sin-Ying Tan, ${ }^{b}$ Grant B. Webber, ${ }^{b,}$ Steven P. Armes, ${ }^{a}$ \\ Seher Ata, ${ }^{c}$ Erica J. Wanless, ${ }^{b, *}$
}

\begin{abstract}
The interactions of two oil droplets grown in the presence of swollen, lightly cross-linked cationic poly(tert-butylamino)ethyl methacrylate (PTBAEMA) microgels was monitored using a high-speed video camera. Three oils ( $n$-dodecane, isopropyl myristate and sunflower oil) were investigated, each in the absence and presence of an oil-soluble cross-linker [tolylene 2,4diisocyanate-terminated poly(propylene glycol), PPG-TDI]. Adsorption of the swollen microgel particles was confirmed by interfacial tension, interfacial elasticity and dilational viscosity measurements on single pendant oil droplets, and assessment of the oscillatory dynamics for coalescing droplet pairs. Like the analogous bulk emulsions, particle adsorption alone did not prevent coalescence of pairs of giant Pickering emulsion droplets. However, prior addition of surface-active PPG-TDI crosslinker to the oil phase results in the formation of highly stable microgel colloidosomes via reaction with the secondary amine groups on the PTBAEMA chains. Colloidosome stability depended on the age of the oil-water interface. This reflects a balance between the adsorption kinetics of the PPG-TDI cross-linker and the microgel particles, each of which must be present at the interface to form a stable colloidosome. Colloidosome formation was virtually instantaneous in $n$-dodecane, but took up to $120 \mathrm{~s}$ in the case of isopropyl myristate. The impact of an acid-induced latex-to-microgel transition on the interaction of giant colloidosomes (originally prepared at $\mathrm{pH} 10$ using isopropyl myristate) was also studied. This acid challenge did not result in coalescence, which is consistent with a closely-related study (A. J. Morse et al., Langmuir, 2014 , 30 (42), 1250912519). No evidence was observed for inter-colloidosome cross-linking, which was attributed to retention of an aqueous film between the adjacent pair of colloidosomes.
\end{abstract}

\section{Introduction}

Colloidosomes are two-phase systems consisting of particlestabilised droplets of one liquid dispersed in a second immiscible liquid phase. ${ }^{1-3}$ They are formed via further stabilisation of precursor Pickering emulsions, which may be prepared using either inorganic or polymeric particles. This shell reinforcement can be achieved by thermal annealing, ${ }^{4-7}$ polyelectrolyte complexation, ${ }^{8,} 9$ gel trapping, ${ }^{10,}{ }^{11}$ polymerisation, ${ }^{12-14}$ or covalent cross-linking, ${ }^{15,16}$ as reviewed recently by Thompson et al. ${ }^{17}$ One advantage of the latter approach is the absence of any heating step, making it an attractive option for the formation of microcapsules containing a cargo of bioactive molecules. In principle, covalent cross-linking can be achieved using reagents located in either the continuous phase ${ }^{18,19}$ or the dispersed phase..$^{20}$ Confining the cross-linking agent to the dispersed phase reduces the probability of inter-colloidosome fusion, enabling colloidosomes to be prepared at relatively high volume fractions. ${ }^{21}$

Stimulus-responsive colloidosomes can be obtained by using microgels to stabilise the Pickering emulsions. For example, thermo-responsive poly $(\mathrm{N}$-isopropylacrylamide- $\mathrm{co}$-acrylic acid) P(NIPAM-co-AA) microgel particles were used by Lawrence et al. to stabilise aqueous droplets dispersed in $n$-octanol. ${ }^{22}$ These physically cross-linked colloidosomes could be subsequently transferred into water and exhibited a significant volume

\footnotetext{
a. Department of Chemistry, University of Sheffield, Brook Hill, Sheffield, South Yorkshire, S3 7HF, United Kingdom.

b. Priority Research Centre for Advanced Particle Processing and Transport, University of Newcastle, Callaghan, NSW, 2308, Australia.

c. School of Mining Engineering, University of New South Wales, Sydney, NSW 2052, Australia.

* Joint corresponding authors: erica.wanless@newcastle.edu.au \& grant.webber@newcastle.edu.au

†Electronic Supplementary Information (ESI) available: Supplementary material and methods, and presentation of data for sunflower oil based systems.
}

reduction upon heating as a result of a coil-to-globule transition. Similarly, chemically cross-linked primary amine-functionalised PNIPAM microgel colloidosomes have been reported by Shah et al., who utilised glutaraldehyde as a cross-linking agent. ${ }^{23}$ This reagent was confined to the aqueous droplet phase and the resulting microcapsules also displayed a substantial volume reduction as the temperature was raised above the LCST of the PNIPAM chains. More recently, $\mathrm{pH}$-responsive colloidosomes have been prepared from oil-in-water Pickering emulsions stabilised by poly(tert-(butylamino)ethyl methacrylate) (PTBAEMA) latexes at $\mathrm{pH} 10 .{ }^{24}$ The latex shell was stabilised using a polymeric diisocyanate cross-linker confined to the oil phase to form stable colloidosomes. ${ }^{25}$ In contrast to the acidinduced demulsification of the analogous Pickering emulsions, the cross-linked colloidosomes survived a switch in the continuous phase $\mathrm{pH}$ from 10 to 3 without any change in the oil droplet size. This indicates that the acid-induced latex-tomicrogel transition is confined to the oil-water interface, and that shell cross-linking is sufficiently robust to produce highly cationic microgel colloidosomes. ${ }^{26}$ In summary, microgel colloidosomes can be formulated at high volume fractions, and remain stable in various environments, thus enabling these capsules to be considered for controlled release applications. ${ }^{17}$ In the above literature examples, the colloidosome size range is typically $10-100 \mu \mathrm{m}$ diameter with individual colloidosome stability being assessed from the integrity of dried colloidosomes via electron microscopy. However, the stability of particlestabilised droplet interfaces can be investigated in situ by increasing the droplet size to the visible range. So-called 'giant' (i.e. millimetre-sized) colloidosomes were first reported by Ashby et al., who successfully produced water droplets stabilised by polystyrene latex via transfer through a planar oil-water interface. ${ }^{27}$ The interaction and coalescence dynamics of two such particle-stabilised interfaces can be investigated using highspeed video imaging as demonstrated by Ata. ${ }^{28}$ This approach 
was used to demonstrate the superior stability of interacting giant colloidosomes compared to the analogous Pickering droplets. ${ }^{29}$ More specifically, poly(glycerol monomethacrylate)-stabilised polystyrene latex particles were adsorbed at the surface of sunflower oil droplets and cross-linking was achieved within 20 min using an oil-soluble polymeric diisocyanate cross-linker. If this cross-linker is introduced into just one of the two interacting droplets then pairwise interactions between giant colloidosomes and giant Pickering droplets can be studied. Evidence was presented for diffusion of the oil-soluble cross-linker from the colloidosome into the adjacent Pickering droplet, which indicated complete drainage of the aqueous film between the contacting droplets.

In a closely-related study, the interaction and stability of pairs of giant colloidosomes and Pickering droplets stabilised by PTBAEMA latexes was investigated. ${ }^{30}$ More rapid cross-linking kinetics was observed in this case, since isocyanates react faster with the secondary amine group on the TBAEMA repeat units than with hydroxyl groups. ${ }^{25}$ However, the rate of adsorption of the PTBAEMA latex particles at the oil-water interface was relatively slow. This resulted in arrested coalescence when studying the asymmetric interaction between a giant colloidosome and a giant Pickering droplet for relatively short ageing times (e.g. $60 \mathrm{~s}$ ), which suggested that insufficient particle adsorption had occurred in the contact zone between the two droplets on this time scale.

The influence of swellable microgel particles adsorbed at fluid interfaces can also be investigated by visual assessment of the stability of giant droplets or bubbles. For example, the stability conferred by sterically-stabilised poly(2-vinylpyridine) (P2VP) particles adsorbed at the air-water interface was assessed via high-speed video imaging studies of interacting pairs of air bubbles in water by Ata et al. ${ }^{31} \mathrm{P} 2 \mathrm{VP}$ particles produced air bubbles that were stable towards coalescence in their latex form at $\mathrm{pH} 10$, but on lowering the solution $\mathrm{pH}$ to 2 the particles underwent a latex-to-microgel transition and the air bubbles subsequently coalesced upon contact. Similar acidification of the solution $\mathrm{pH}$ for P2VP latex-stabilised air bubbles in contact also resulted in coalescence, suggesting desorption of the swollen P2VP microgel particles from the air/water interface.

Herein we investigate the formation, interaction dynamics and stability towards coalescence of pairs of giant colloidosomes formed by swollen cross-linkable PTBAEMA microgel particles (at $\mathrm{pH} 3$ ) for three different oils, namely $n$-dodecane, isopropyl myristate or sunflower oil. The particles are swollen due to protonation of the secondary amine residues on the PTBAEMA chains. This study is the first to assess the stability of such Pickering droplets or colloidosomes at $\mathrm{pH} \mathrm{3,} \mathrm{as} \mathrm{the} \mathrm{swollen}$ PTBAEMA microgel particles do not form stable Pickering emulsions, or therefore colloidosomes, under high shear homogenisation. ${ }^{24}$ The oils were chosen for comparison to our previous work examining PTBAEMA latex stabilised Pickering emulsions and colloidosomes prepared by high shear homogenisation ${ }^{24,25}$ and our study of the interactions of pairs of giant Pickering droplets and colloidosomes stabilised by PTBAEMA latex particles at $\mathrm{pH} 10 .^{30} \mathrm{We}$ also examine the impact of an acid-induced latex-to-microgel transition on the pairwise interaction of these giant colloidosomes, which we have previously reported does not cause demulsification of the bulk emulsion. ${ }^{25}$

\section{Experimental}

\section{Materials}

See ESI Materials and methods. $\dagger$

\section{Aqueous emulsion polymerisation}

See ESI Materials and methods. $\uparrow$

Dynamic Light Scattering (DLS)

See ESI Materials and methods. $\dagger$

\section{Aqueous Electrophoresis}

See ESI Materials and methods. $\dagger$

\section{Droplet coalescence apparatus}

The apparatus used in this work has been previously described for studying the coalescence of pairs of latex-coated air bubbles ${ }^{31}$ or oil droplets ${ }^{29,30}$ in aqueous solution. Two stainless steel capillaries $(0.41 \mathrm{~mm}$ inner diameter and $0.71 \mathrm{~mm}$ outer diameter $)$ were inserted into a beaker containing $50 \mathrm{~mL}$ aqueous latex dispersion and a magnetic stirrer bar. Two micro-syringe pumps (Sarasota, FL) were used to produce $n$-dodecane, isopropyl myristate or sunflower oil droplets from the tips of the capillaries. Between experiments, the glassware, syringes, capillaries and stirrer bar were thoroughly cleaned using 2propanol or ethanol to remove oil residue and then rinsed with Millipore Milli-Q water at least ten times to remove residual contaminants. Coalescence data were recorded using a highspeed video camera (Phantom 5, vision research) at a rate of up to 6024 frames $\mathrm{s}^{-1}$. Images were collected using Phantom 6.30 (Nikon) software at $512 \times 256$ resolution. A light source was used for back illumination of the droplets.

\section{Sample Preparation}

The latex dispersions used in this work were required to be of sufficiently low turbidity to enable high-speed video capture of high-quality images. A $240 \pm 50 \mathrm{~nm}$ diameter PTBAEMA latex $(0.20 \mathrm{~mL}$ of a $3.9 \mathrm{wt} \%$ dispersion) was added to a beaker containing $50 \mathrm{~mL}$ of $0.01 \mathrm{M} \mathrm{KNO}_{3}$ (or $0.1 \mathrm{M}$ for the sunflower oil experiments) solution at $\mathrm{pH} 10$. These minimum concentrations of added electrolyte required to promote bubble instability were used throughout. ${ }^{30}$ The final latex concentration was $1.6 \times 10^{-2} \mathrm{wt} \%$.

\section{Droplet coalescence protocol, data analysis, acid challenge and cross-linking experiments}

The required oil was held in the two syringes and then connected to the capillaries. All air bubbles were expelled from the capillaries. The beaker containing the PTBAEMA latex dispersion and a magnetic flea (or without the addition of particles for the bare oil droplet experiments) was placed inside a rectangular Perspex vessel containing water to prevent optical distortion arising from the cylindrical surface of the beaker. The 
beaker was then placed on a magnetic stirrer mounted on a lab jack beneath the capillaries. $2 \mathrm{~mL}$ of the oil used was injected on to the top of the water surface once the capillaries were submerged in the solution. This was required to maintain a constant surface area of the oil throughout the experiment, as well as preventing evaporation of the aqueous phase. Oil droplets were then grown from the capillaries until the droplets were 1.8 $2.0 \mathrm{~mm}$ in diameter. During droplet ageing the aqueous phase was constantly stirred. The capillaries were moved together using a linear actuator at a speed of $0.238 \mathrm{~cm} \mathrm{~s}^{-1}$ until the droplets were just touching (a capillary separation of 1.0-1.3 mm depending on the initial droplet diameter). The droplet coalescence time was measured from the moment of droplet contact until coalescence and analysed from the video footage at 300 frames $\mathrm{s}^{-1}$. Each coalescence experiment was conducted at least 10 times and the mean and standard deviation for the coalescence time was recorded. The frame rate was also increased to 6024 frames $\mathrm{s}^{-1}$ to allow detailed analysis of the post-coalescence behaviour. The projected surface area of the droplets before and after coalescence was measured as a function of time. The droplet area in each frame was analysed using Image $\mathbf{J}$ software and the results were plotted as the percentage change in projected area relative to the initial area of the droplets prior to coalescence.

The majority of the oil droplet interaction experiments were performed in aqueous solution at $\mathrm{pH} 3$ (adjusted using $0.10 \mathrm{M}$ $\mathrm{HNO}_{3}$ ). Such experiments were conducted both in the presence of the PTBAEMA particles and in their absence. This was in order to establish whether the swollen microgel particles conferred stabilisation towards coalescence. A second 'acid challenge' approach was to form the oil droplets at $\mathrm{pH} 10$, allow PTBAEMA latex particles to adsorb at the oil-water interface, and then rapidly adjust the $\mathrm{pH}$ to 3 .

The same protocol was also used for the production of covalently cross-linked colloidosomes. In this case, pure oil was replaced with a dilute solution of PPG-TDI cross-linker in the desired oil at a concentration of either $0.1 \mathrm{mg} \mathrm{mL}^{-1}$ (n-dodecane and isopropyl myristate) or $1.0 \mathrm{mg} \mathrm{mL}^{-1}$ (sunflower oil). A higher cross-linker concentration was utilised for the latter highviscosity oil to increase the rate of diffusion of the PPG-TDI in this medium. Colloidosomes were formed in aqueous solution at $\mathrm{pH} 10$, before the solution $\mathrm{pH}$ was lowered to 3 . Then further oil droplets were grown at $\mathrm{pH} 3$ to assess the formation and stability of the microgel colloidosomes.

\section{Pendant drop tensiometry}

See ESI Materials and methods. $\dagger$

\section{Oil droplet zeta potential measurements}

See ESI Materials and methods. $\dagger$

\section{Results and Discussion}

The behaviour of Pickering droplets and colloidosomes of $n$ dodecane and sunflower oil are qualitatively similar aside from the influence of the higher sunflower oil viscosity, though isopropyl myristate exhibited significant differences. We thus concentrate on the results for the $n$-dodecane and isopropyl myristate systems, while data for sunflower oil is presented in the ESI. $\uparrow$

\section{Bulk aqueous solution behaviour of PTBAEMA particles}

The variation in mean hydrodynamic diameter and zeta potential for a $0.01 \mathrm{wt} \%$ aqueous dispersion of PTBAEMA particles as a function of $\mathrm{pH}$ is shown in Figure $\mathrm{S} 1$ of the ESI. $\uparrow$ Above $\mathrm{pH} 8$, the hydrodynamic latex diameter indicated by dynamic light scattering was $240 \pm 50 \mathrm{~nm}$. A latex-to-microgel transition was observed at around $\mathrm{pH}$ 7-8. The cationic microgel particles below $\mathrm{pH} 7$ had a mean diameter of $655 \pm 40 \mathrm{~nm}$, which corresponds to volumetric swelling by a factor of approximately 20 on addition of acid. The zeta potential varied from $-31 \mathrm{mV}$ at $\mathrm{pH} 10$ to $+35 \mathrm{mV}$ at $\mathrm{pH} 3$. The isoelectric point was determined to be $\mathrm{pH} 8.5$, which is between the $\mathrm{p} K$ a for free PTBAEMA chains in dilute solution and the isoelectric point for PEGMAstabilised PTBAEMA latex particles recently reported by Morse and co-workers. ${ }^{24}$

\section{Bare oil droplet interactions at pH 3}

Initially, pure oil droplets prepared at $\mathrm{pH} 3$ in the presence of added salt were investigated to provide suitable 'baseline' behaviour. The zeta potential of these oil droplets was measured to be almost zero $(<10 \mathrm{mV})$. This is in good agreement with an isoelectric point of $\mathrm{pH} 2.1$ reported for $n$-dodecane. ${ }^{32}$ In contrast, highly anionic zeta potentials (greater than $-90 \mathrm{mV}$ ) were determined at $\mathrm{pH} 10$, which have been attributed to adsorption of hydroxide ions from solution. ${ }^{33,} 34$ The dynamic interfacial tension calculated from pendant drop profiles for $n$-dodecane and isopropyl myristate at $20{ }^{\circ} \mathrm{C}$ are shown in Figure 1 (sunflower oil droplets in Figure S2 of ESI†). 
(A)

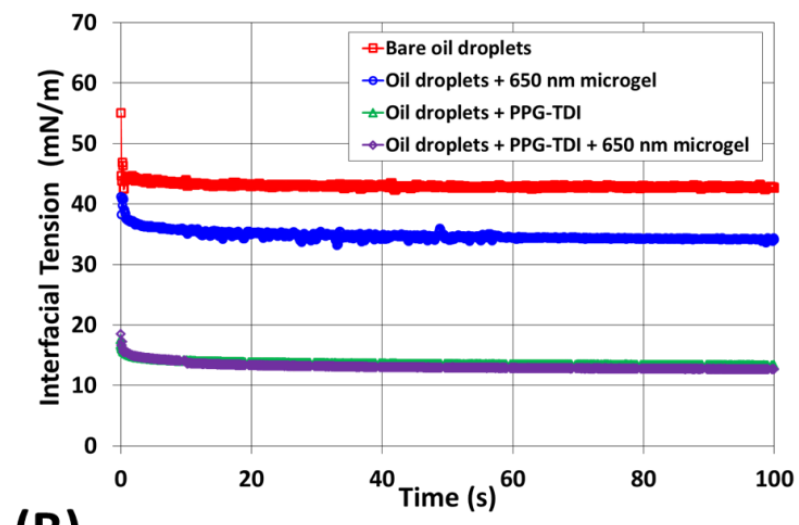

(B)

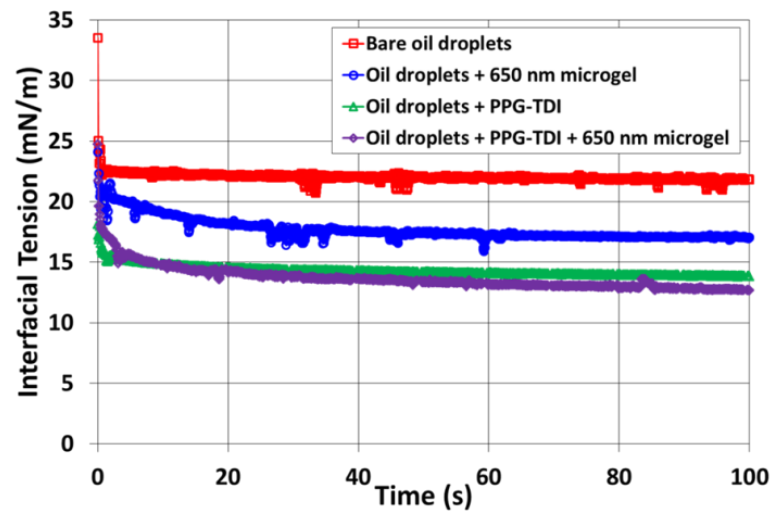

Fig. 1. Dynamic interfacial tension of (A) $n$-dodecane and (B) isopropyl myristate in the absence $(\square)$ and presence (O) of a $3.8 \times 10^{-2} \% \mathrm{w} / \mathrm{v}$ aqueous dispersion of charge-stabilised PTBAEMA microgel particles, as well as bare oil droplets in the presence of an oil-soluble cross-linker, PPG-TDI $(\Delta)$ and oil droplets prepared in the presence of both PTBAEMA microgel particles and PPG-TDI $(\diamond)$. The aqueous phase was $\mathrm{pH} 3$ and $0.01 \mathrm{M} \mathrm{KNO}_{3}$ background electrolyte in each case.

The mean values for droplets aged for 5 min are given in Table 1 and Table S1. The occasional vertical blips in the data are attributed to a temporary loss of image resolution and are experimental artefacts. The $n$-dodecane/water interface rapidly attained equilibrium within $15 \mathrm{~s}$, whereas the isopropyl myristate oil-water interface required $\sim 5 \mathrm{~min}$ to achieve its equilibrium interfacial tension. The interfacial tension of the $n$ dodecane/water interface after ageing for $5 \mathrm{~min}$ is twice that of isopropyl myristate. Each interfacial tension is higher than that previously measured at $\mathrm{pH} 10 .{ }^{30}$ In addition, the interfacial tension of each oil containing the PPG-TDI cross-linker was measured in the presence of added salt at $\mathrm{pH} 3$. This reagent has been shown to be highly surface-active in $n$-dodecane, but only weakly surface-active in isopropyl myristate at $\mathrm{pH} 10 .^{30}$ The rapid reduction to substantially lower interfacial tensions for both oils at $\mathrm{pH} 3$ shown in Figure 1 suggests high surface activity under these conditions.

Droplet coalescence was observed at $\mathrm{pH} 3$ in the presence of added salt, with mean coalescence times for pairs of interacting oil droplets summarised in Table 1 and Table S1. The $n$ dodecane and isopropyl myristate droplet stabilities were similar
Table 1. Influence of oil type and presence of oil-soluble cross-linker (PPG-TDI) on the interfacial tension, coalescence time and damping coefficient of pendant oil droplets grown in aqueous $0.01 \mathrm{M} \mathrm{KNO}_{3}$ background electrolyte at $\mathrm{pH}$ 3. Oil droplets were 1.8 to $2.0 \mathrm{~mm}$ in diameter.

\begin{tabular}{c|c|c|c}
\hline \multirow{2}{*}{ Interface } & $\begin{array}{c}\text { Interfacial } \\
\text { Tension After } \\
5 \mathrm{~min} \\
\text { Equilibration } \\
\left(\mathrm{mN} \mathrm{m}^{-1}\right)\end{array}$ & $\begin{array}{c}\text { Coalescence } \\
\text { Time }(\mathrm{s})\end{array}$ & $\begin{array}{c}\text { Damping } \\
\text { Coefficient } \\
\left(\mathrm{ms}^{-1}\right)\end{array}$ \\
\hline$n$ - & & $0 \mathrm{~s}$ ageing: & $0.0426 \pm$ \\
Dodecane/aqueous & $43.1 \pm 0.3$ & $8.0 \pm 0.8$ & 0.008 \\
solution & & $60 \mathrm{~s}$ ageing: & $0.0418 \pm$ \\
(n-Dodecane + & & $8.7 \pm 0.9$ & 0.003 \\
\hline PPG-TDI)/aqueous & $13.1 \pm 0.1$ & $20.8 \pm 3.1$ & 0.008 \\
solution & & $60 \mathrm{~s}$ ageing: & $0.0523 \pm$ \\
Isopropyl & & $35.7 \pm 8.4$ & 0.002 \\
\hline myristate/aqueous & $21.3 \pm 0.3$ & $0 \mathrm{~s}$ ageing: & $0.0409 \pm$ \\
solution & & $60 \mathrm{~s}$ ageing: & $0.0407 \pm$ \\
& & $8.4 \pm 4.2$ & 0.002 \\
\hline (Isopropyl & & $0 \mathrm{~s}$ ageing: & $0.0407 \pm$ \\
myristate + PPG- & $13.9 \pm 0.8$ & $11.9 \pm 3.4$ & 0.006 \\
TDI)/aqueous & & $60 \mathrm{~s}$ ageing: & $0.0412 \pm$ \\
solution & & $12.2 \pm 2.7$ & 0.003 \\
\hline
\end{tabular}

and there was no significant difference in the coalescence time if the droplets were aged for $60 \mathrm{~s}$ prior to contact. Upon coalescence, the resulting single droplet exhibited dynamic oscillatory behaviour until the excess energy was dissipated into the aqueous phase. Frame-by-frame analysis of video recordings of such coalescence events was conducted by plotting the change in the projected surface area as a function of time, as shown in Figure 2 (ESI $\dagger$ Figure S3 for sunflower oil). The oscillatory profiles can be fitted to a damped simple harmonic oscillator including a suitable damping coefficient. ${ }^{35}$ The damping coefficients in Table 1 are quite similar for both oils suggesting their similar bulk viscosities are dominating the post-coalescence behaviour.

For all oils the mean coalescence time was longer in the presence of PPG-TDI. This is consistent with adsorption of this crosslinker and the concomitant lowering of the interfacial tension, which reflects a lower energy per unit area and a greater resistance towards coalescence. The substantial further increase in coalescence time when the $n$-dodecane droplets were initially aged for $60 \mathrm{~s}$ suggests strong adsorption of PPG-TDI at this oil interface, which is consistent with the large reduction in interfacial tension shown in Figure 1. The intrinsic surface activity of the PPG-TDI also contributed to higher damping coefficients for all three oils, with additional increases being observed for aged droplets. This is consistent with an earlier report of surfactant adsorption resulting in enhanced damping of the post-coalescence dynamics. ${ }^{36}$ 


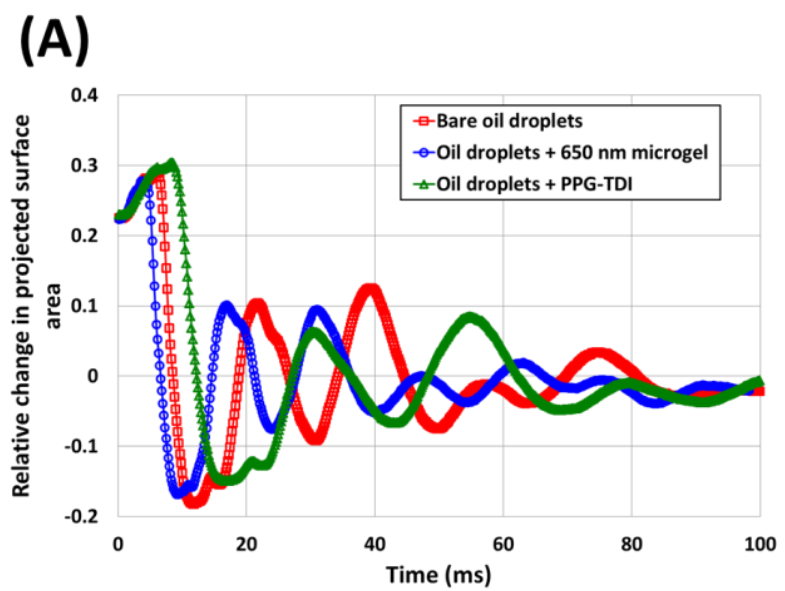

(B)

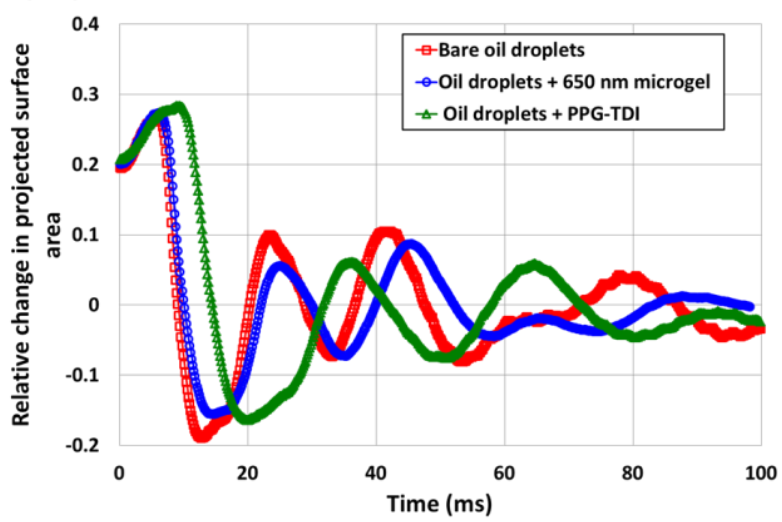

Fig. 2. Comparison of the coalescence dynamics of pairs of $(A) n$-dodecane and (B) isopropyl myristate droplets in the absence ( $\square$ ) and presence (O) of a $1.6 \times 10^{-2}$ $w t \%$ aqueous dispersion of $655 \mathrm{~nm}$ PTBAEMA microgel particles, as well as bare oil droplets in the presence of an oil-soluble cross-linker, PPG-TDI $(\Delta)$. Al experiments were conducted at $\mathrm{pH} 3$ and $0.01 \mathrm{M} \mathrm{KNO}_{3}$ background electrolyte.

\section{Pickering droplet interactions at $\mathrm{pH} 3$}

It is worth briefly commenting on the giant PTBAEMAstabilised Pickering droplets that have been previously prepared at $\mathrm{pH} 10 .^{30}$ The charge-stabilised PTBAEMA latex was adsorbed onto pairs of oil droplets of either $n$-dodecane, isopropyl myristate or sunflower oil to form giant Pickering droplets. When these Pickering droplets were brought into close contact, coalescence occurred for all three oils. Thus the adsorbed latex particles do not confer stability on millimetre-sized droplets, despite their efficient Pickering emulsifier stabilisation of much finer emulsion droplets prepared via high shear homogenisation. ${ }^{24}$ This striking difference in droplet stability was attributed to the much more highly curved contact zones between droplets in the conventional emulsion. ${ }^{30}$ Nevertheless, greater damping for each oil upon coalescence of the giant Pickering droplets (relative to bare oil droplet coalescence at $\mathrm{pH}$ 10) confirmed particle adsorption at each oil/water interface.

When analogous experiments were performed at $\mathrm{pH} 3$ in the presence of swollen microgel particles, coalescence was also observed for droplet pairs of all oils brought into contact approximately $10-15 \mathrm{~s}$ after formation, as shown in Figure 3

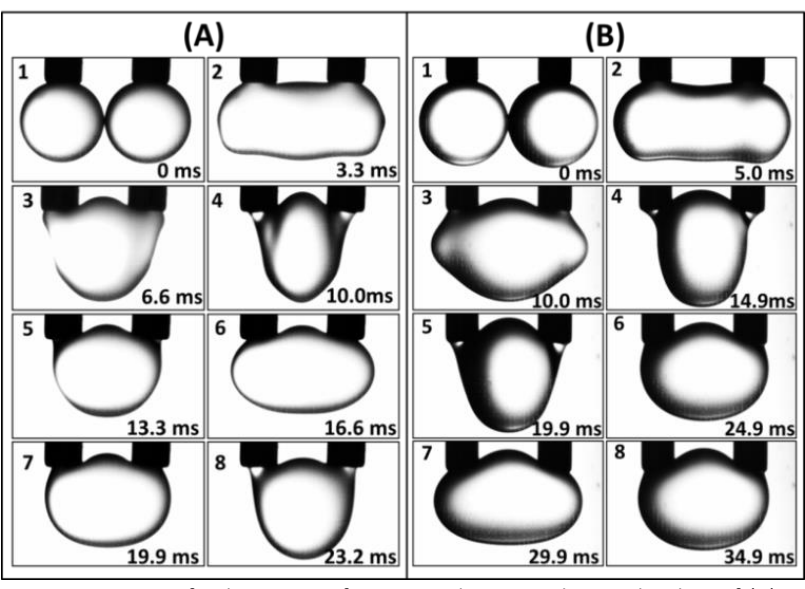

Fig. 3. Sequence of video images for two coalescing Pickering droplets of (A) ndodecane (images shown for every twentieth frame) and (B) isopropyl myristate (images shown for every thirtieth frame) in the presence of a $1.6 \times 10-2 \mathrm{wt} \%$ aqueous dispersion of $655 \mathrm{~nm}$ charge-stabilised PTBAEMA microgel particles at $\mathrm{pH}$ 3 and $0.01 \mathrm{M} \mathrm{KNO}_{3}$ background electrolyte. The outer diameter of the capillaries is $0.71 \mathrm{~mm}$, which acts as an appropriate scale bar.

(ESI† Figure S4 for sunflower oil). This is consistent with the observation that no stable Pickering emulsion droplets are obtained when high shear homogenisation is conducted at $\mathrm{pH}$ $3{ }^{25}$ However, the dynamic interfacial tension profiles presented in Figure 1 and the data shown in Table 2 suggest that some microgel particles do adsorb at the oil-water interface from acidic solution $(\mathrm{pH} 3)$, but the reduction in interfacial tension is far less significant than that observed for the corresponding latex particles at $\mathrm{pH} 10 .^{30}$ This indicates a much lower driving force for adsorption, which is consistent with the near-neutral zeta potentials observed for the oil droplets at $\mathrm{pH} 3$. Importantly, such adsorption requires about $60 \mathrm{~s}$ in both $n$-dodecane and isopropyl myristate. The largest reduction in interfacial tension is for $n$ dodecane.

The coalescence times for oil droplet pairs displayed in Table 2 also indicate a change in the nature of the oil-water interfaces in the presence of the microgel particles at $\mathrm{pH} 3$. The coalescence times observed in the absence of any droplet ageing prior to contact were shorter for both oils compared to those observed for the bare oil interfaces. A small increase in stability was also observed with ageing time for the case of interacting $n$-dodecane droplets (an effect not investigated in the case of isopropyl myristate). These coalescence times are accompanied by changes in the damping coefficients of the post-coalescence oscillation dynamics relative to the bare oil interfaces. Greater damping was observed for both oils compared to the respective pure oil drops (Table 1). This is consistent with the presence of a surface-active microgel coating adsorbed at the interface, ${ }^{36-38}$ but in principle could also reflect an increase in viscosity for the surrounding aqueous solution. However, this is unlikely at such a low particle concentration since, for a similar system, we detected no increase in the bulk solution viscosity. ${ }^{31}$

The lower interfacial tensions, enhanced oil droplet stabilities and greater damping coefficients observed for both oils suggest microgel adsorption at the near-neutral oil/water interfaces to form giant Pickering microgel droplets. However, such 
Table 2. Influence of oil type and presence of oil-soluble cross-linker (PPG-TDI) on the interfacial tension, coalescence time and damping coefficient of pendant oi droplets grown in the presence of $655 \mathrm{~nm}$ charge-stabilised PTBAEMA microgels in aqueous $0.01 \mathrm{M} \mathrm{KNO}_{3}$ background electrolyte at $\mathrm{pH} 3$. Oil droplets were 1.8 to $2.0 \mathrm{~mm}$ in diameter.

\begin{tabular}{|c|c|c|c|}
\hline Interface & $\begin{array}{c}\text { Interfacial } \\
\text { Tension } \\
\text { After } 5 \text { min } \\
\text { Equilibration } \\
\quad\left(\mathrm{mN} \mathrm{m}^{-1}\right) \\
\end{array}$ & $\begin{array}{c}\text { Coalescence } \\
\text { time (s) }\end{array}$ & $\begin{array}{l}\text { Damping } \\
\text { coefficient } \\
\left(\mathrm{ms}^{-1}\right)\end{array}$ \\
\hline $\begin{array}{c}n- \\
\text { Dodecane/aqueous } \\
\text { solution }\end{array}$ & $33.2 \pm 1.6$ & $\begin{array}{l}0 \text { s ageing: } \\
1.26 \pm 0.6 \\
60 \text { s ageing: } \\
1.88 \pm 0.4\end{array}$ & $\begin{array}{c}0.0560 \pm \\
0.008 \\
0.0533 \pm \\
0.001\end{array}$ \\
\hline $\begin{array}{l}\text { (n-Dodecane + } \\
\text { PPG-TDI)/aqueous } \\
\text { solution }\end{array}$ & $12.3 \pm 0.1$ & $\begin{array}{c}0 \text { s ageing: } \\
\text { No } \\
\text { coalescence } \\
60 \text { s ageing: } \\
\text { No } \\
\text { coalescence }\end{array}$ & $\begin{array}{c}\text { No } \\
\text { coalescence } \\
\text { No } \\
\text { coalescence }\end{array}$ \\
\hline $\begin{array}{c}\text { Isopropyl } \\
\text { myristate/aqueous } \\
\text { solution }\end{array}$ & $16.7 \pm 0.5$ & $\begin{array}{l}0 \text { s ageing: } \\
4.7 \pm 0.5\end{array}$ & $\begin{array}{c}0.0500 \pm \\
0.008\end{array}$ \\
\hline $\begin{array}{c}\text { (Isopropyl } \\
\text { myristate + PPG- } \\
\text { TDI)/aqueous } \\
\text { solution }\end{array}$ & $11.9 \pm 0.1$ & $\begin{array}{c}0 \text { s ageing: } \\
3.4 \pm 0.7 \\
60 \text { s ageing: } \\
54 \pm 17 \\
120 \text { s ageing: } \\
\text { n - }\end{array}$ & $\begin{array}{c}0.0485 \pm \\
0.008 \\
0.0462 \pm \\
0.008 \\
\text { No }\end{array}$ \\
\hline
\end{tabular}

adsorption is expected to be weak, with the potential for interfacial mobility. Indeed, the data shown in Table 3 indicate an increase in interfacial elasticity for $n$-dodecane and sunflower oil (ESI† Table S2) droplets in the presence of microgel particles (in contrast, the interfacial elasticity for isopropyl myristate is constant within experimental uncertainty). The simultaneous reduction in dilational viscosity suggests that the interface is less able to respond rapidly to changes in interfacial area because of the presence of adsorbed particles; the adsorption or desorption of particles is relatively slow. ${ }^{39}$ Interfacial elasticity and dilational viscosity derive from the response of interfacial tension of a droplet to sinusoidal changes in its volume; the elasticity measures the magnitude of the response and the viscosity how rapidly it responds. Analysis of the diffusion limited adsorption of polymeric microgels at the oil/water interface using the Ward-Tordai method shows the interfacial diffusion coefficients can be very small. ${ }^{40,} 41$ Adsorbed PNIPAM-based microgels may also undergo deformation and flattening. ${ }^{40,42,43}$ In these systems, significant overlap between adjacent adsorbed deformed microgel particles was reported. ${ }^{42}$ However, these PNIPAM microgels were essentially uncharged, whereas the PTBAEMA microgels studied herein are highly cationic and therefore unlikely to overlap during their adsorption; that is, the microgels studied here remain as discrete objects at the interface.

\section{Colloidosome vs colloidosome interactions at pH 3}

It is instructive to consider the giant PTBAEMA-based colloidosomes that have been previously prepared at $\mathrm{pH} 10 .{ }^{30} \mathrm{In}$ this earlier study, droplet coalescence was prevented by crosslinking adjacent PTBAEMA latex particles at the oil-water interface via addition of PPG-TDI to the oil phase. It is emphasised that minimal droplet ageing was required to produce stable millimetre-sized colloidosomes under these conditions (just 10-15 s is needed to grow two droplets and move them into contact), which indicates that the rate of cross-linking is rapid. These observations mirror those made for the corresponding 140-240 $\mu \mathrm{m}$ diameter colloidosomes prepared from Pickering emulsions generated via high shear homogenisation. ${ }^{25}$ In addition to exhibiting enhanced stability towards coalescence, inter-colloidosome cross-linking was observed when the giant colloidosomes were in contact, suggesting diffusion of the oilsoluble cross-linker across the inter-droplet contact zone. This was evident in the form of a cross-linked 'neck' between the two adjacent giant colloidosomes upon separation, indicating complete drainage of the intervening aqueous film.

In the present study, the PPG-TDI cross-linker was added to the oil droplets prepared at $\mathrm{pH} 3$ in order to assess how cross-linking any adsorbed microgel particles at the oil-water interface might affect droplet stability. A schematic of this situation is shown in Figure 4. This investigation has no parallel in the bulk, since Pickering emulsions could not be formed at $\mathrm{pH} 3$ when using cationic swollen PTBAEMA microgel particles. ${ }^{24}$ In contrast, interactions between pairs of giant microgel colloidosomes could be investigated. Interestingly, the oils differed considerably in their behaviour, with a second important parameter being the age of the interface (defined as the total time prior to the droplets being moved into contact, with the latter protocol requiring an additional 10-15 s). The results are summarised in Table 2.

Interacting pairs of $n$-dodecane droplets containing $0.1 \mathrm{mg} \mathrm{mL}^{-1}$ PPG-TDI cross-linker rapidly formed highly stable giant microgel colloidosomes, since no coalescence was observed after $5 \mathrm{~min}$ in contact regardless of whether the droplets were moved into contact immediately, or aged for $60 \mathrm{~s}$ apart and then moved into contact, see Figure 5A. This is consistent with the rapid reduction and equilibration of the interfacial tension shown in Figure 1 in the presence of both PPG-TDI cross-linker and the microgel particles. In addition, $n$-dodecane droplet pairs forced into greater contact (Figure 5A, Image 5) proved to be stable towards coalescence and could be subsequently separated intact. This stability indicates efficient cross-linking which, while confined to the oil phase (and therefore most likely not affected by the aqueous phase $\mathrm{pH}$ ), confirms the adsorption of PTBAEMA microgel particles at the $n$-dodecane/water interface and colloidosome formation. There was no evidence for PPGTDI diffusion between pairs of giant microgel colloidosomes prepared using $n$-dodecane. This suggests the retention of an aqueous film between the droplets when brought into contact, promoted by the presence of highly swollen adsorbed microgel particles. This is in contrast to colloidosomes formed at $\mathrm{pH} 10 .{ }^{30}$ 


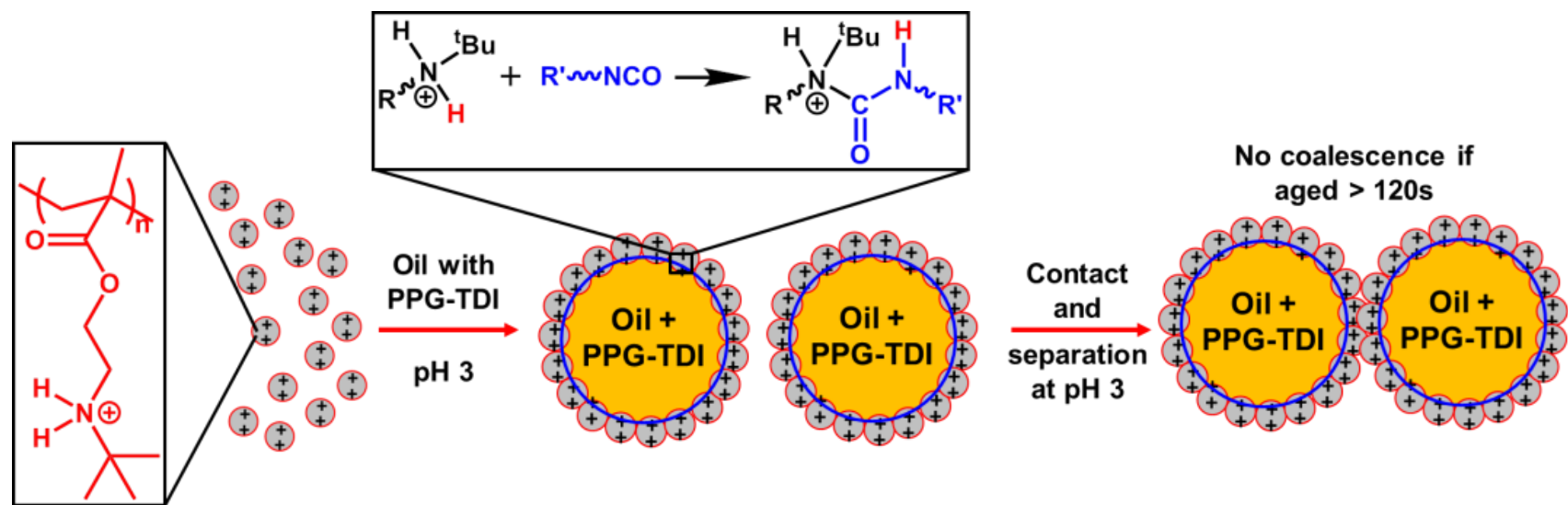

Fig. 4. Reaction scheme for the formation of covalently cross-linked microgel colloidosomes, which were formed when oil ( $n$-dodecane, isopropyl myristate or sunflower oil) droplets containing PPG-TDI cross-linker were exposed to a $1.6 \times 10^{-2} \mathrm{wt} \%$ aqueous dispersion of charge-stabilised PTBAEMA latex at pH 3. Microgel colloidosomes that were aged $>120 \mathrm{~s}$ did not coalesce and could be separated following contact.

The isopropyl myristate droplet pairs containing $0.1 \mathrm{mg} \mathrm{mL}-1$ PPG-TDI exhibited qualitatively different behaviour that depended on the droplet ageing time. This is perhaps expected based on the long equilibration time (>120 s) of the interfacial tension indicated in Figure 1, which suggests simultaneous adsorption of both PPG-TDI cross-linker and the microgel particles. If such droplet pairs were brought into contact immediately upon formation, coalescence was observed. In fact, the coalescence time was slightly shorter than that reported for the Pickering system in the absence of any PPG-TDI, see Table 2. Two opposing effects are operating here: cross-linker adsorption promotes stability as shown in Table 1, but microgel adsorption reduces stability, see Table 2 . However, when the two isopropyl myristate droplets were aged while apart for $60 \mathrm{~s}$ prior to being moved into contact, significantly greater stability was evident as shown in Table 2. Although such droplets still undergo coalescence, this provides strong evidence for microgel adsorption and the onset of cross-linking. ${ }^{29}$ Further particle adsorption is also possible during the ageing period as suggested by Figure 1, although there was no significant change in the postcoalescence damping coefficient for ageing times of 0-60 s. Further ageing of the isopropyl myristate droplets for $120 \mathrm{~s}$ prevented coalescence upon contact. Moreover, when these microgel colloidosomes were subsequently moved apart after 5 min in contact, a narrow 'neck' was formed between them (see Figure 5B, Images 4-6). Such deformation indicates intercolloidosome cross-linking. Indeed, upon further increasing the separation distance, the right-hand colloidosome became detached from its needle rather than from its neighbouring colloidosome. The interaction of sunflower oil colloidosomes is shown in Figure S5 (ESI $\dagger$ ).

The symmetric interaction of two giant droplets containing PPGTDI while also in contact with aqueous dispersions of swollen microgel particles confirms adsorption of this cross-linker at each oil-water interface. Under these conditions,

coalescence is prevented by the cross-linked microgel particles adsorbed onto each droplet, as shown schematically in Figure 4. Swollen microgel particles may continue to adsorb and reinforce the interface during ageing of the two contacted droplets, apart from within the contact zone itself. Interestingly, a reduction in interfacial elasticity is observed for all oils when PPG-TDI is present, see Table 3 and Table S2. This is evident regardless of whether particles are also adsorbed at the interface. This suggests that, in the case of the colloidosomes, unreacted PPG-TDI further stabilises the interface as it is more resilient to changes in drop volume. Moreover, the dilational viscosity data summarised in Table 3 and Table S2 confirms reinforcement of the interface. ${ }^{44}$, 45 This reinforcement is likely a combination of inter-particle and intra-particle cross-linking although the final distribution of the cross-linker cannot readily be determined.

\section{Colloidosome vs colloidosome interactions when aged in isolation at $\mathrm{pH} 10$ prior to a $\mathrm{pH}$ jump to $\mathrm{pH} 3$}

The structural integrity of PTBAEMA colloidosomes formed via high shear homogenisation of $n$-dodecane at $\mathrm{pH} 10$ when subjected to a so-called 'acid challenge' has been previously reported by Morse and co-workers. ${ }^{25}$ This stability confirms effective interfacial cross-linking of the adsorbed latex particles at $\mathrm{pH} 10$, which suggests that hindered microgel swelling

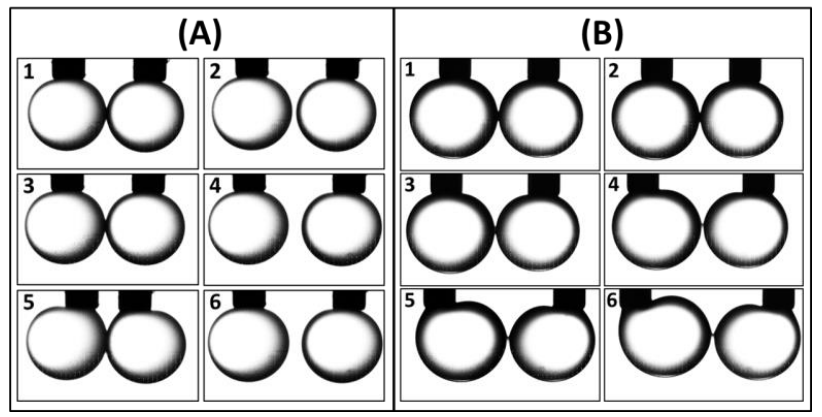

Fig. 5. Sequence of images for two pendant microgel colloidosomes at $\mathrm{pH} 3$ showing contact and separation. The colloidosomes were formed when (A) $n$ dodecane or (B) isopropyl myristate oil droplets containing PPG-TDI cross-linker ( $0.1 \mathrm{mg} \mathrm{mL}^{-1}$ ) were exposed to a $1.6 \times 10^{-2}$ wt\% aqueous dispersion of $655 \mathrm{~nm}$ charge-stabilised PTBAEMA microgel particles with $0.01 \mathrm{M} \mathrm{KNO}_{3}$ background electrolyte. The droplets were aged in isolation for $60 \mathrm{~s}$ (n-dodecane) or $120 \mathrm{~s}$ (isopropyl myristate) before being moved into contact (Image 1). After 5 min contact, the droplets were moved apart stepwise using a linear actuator with a step size of $0.15 \mathrm{~mm}$ (Images 2-6). In A the droplets were brought back into contact following separation. In B, some evidence of inter-colloidosome cross-linking prevented full separation of droplets. 
Table 3. Dynamic interfacial rheological parameters of giant pendant oil droplets grown in both the absence and presence of $655 \mathrm{~nm}$ charge-stabilised PTBAEMA microgels in aqueous $0.01 \mathrm{M} \mathrm{KNO}_{3}$ background electrolyte at $\mathrm{pH} 3$ after ageing for $5 \mathrm{~min}$.

\begin{tabular}{|c|c|c|c|c|}
\hline Interface & $\begin{array}{l}\text { Interfacial } \\
\text { Elasticity } \\
\left(\mathrm{mN} \mathrm{m}^{-1}\right)\end{array}$ & 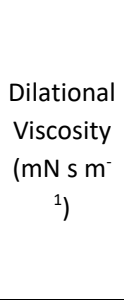 & $\begin{array}{c}\text { Interfacial } \\
\text { Elasticity } \\
\left(\mathrm{mN} \mathrm{m}^{-1}\right) \\
+ \\
\text { PTBAEMA } \\
\text { microgel } \\
\text { Particles }\end{array}$ & $\begin{array}{c}\text { Dilational } \\
\text { Viscosity } \\
\text { (mN s m } \\
1 \text { ) } \\
+ \\
\text { PTBAEMA } \\
\text { microgel } \\
\text { Particles }\end{array}$ \\
\hline $\begin{array}{c}n- \\
\text { Dodecane/aqueous } \\
\text { solution }\end{array}$ & $6.4 \pm 5.6$ & $\begin{array}{c}11.3 \pm \\
3.9\end{array}$ & $13.0 \pm 1.5$ & $4.4 \pm 1.1$ \\
\hline $\begin{array}{c}\text { ( } n \text {-Dodecane + } \\
\text { PPG-TDI)/aqueous } \\
\text { solution }\end{array}$ & $4.9 \pm 0.3$ & $2.4 \pm 0.1$ & $6.2 \pm 0.3$ & $2.2 \pm 0.1$ \\
\hline $\begin{array}{c}\text { Isopropyl } \\
\text { myristate/aqueous } \\
\text { solution } \\
\end{array}$ & $8.5 \pm 0.3$ & $1.1 \pm 0.5$ & $8.5 \pm 1.2$ & $0.7 \pm 0.2$ \\
\hline $\begin{array}{c}\text { (Isopropyl } \\
\text { myristate + PPG- } \\
\text { TDI)/aqueous } \\
\text { solution }\end{array}$ & $6.6 \pm 0.7$ & $3.1 \pm 0.6$ & $7.3 \pm 0.3$ & $1.8 \pm 0.1$ \\
\hline
\end{tabular}

probably occurs at low $\mathrm{pH}$. In addition, the stabilities of interacting pairs of giant PTBAEMA latex colloidosomes prepared using $n$-dodecane, isopropyl myristate or sunflower oil at $\mathrm{pH} 10$ have been reported, as discussed above. ${ }^{30}$ Thus we decided to subject giant colloidosomes prepared at $\mathrm{pH} 10$ to the addition of acid in order to assess their stability after such a $\mathrm{pH}$ jump.

In Figure 6, the change in interfacial tension of a pendant isopropyl myristate Pickering droplet is compared with that of a colloidosome when each was exposed to a $\mathrm{pH}$ jump from 10 to 3. It is clear that the Pickering droplet responds with a rapid increase in interfacial tension while the colloidosome does not respond at all. The former behaviour reflects the greater interfacial tension of an equilibrated Pickering droplet at $\mathrm{pH} 3$ (Table 2) compared to that at $\mathrm{pH} 10 .{ }^{30}$ This change is attributed to the rapid desorption of swollen microgel particles upon exposure to acid followed by the relatively slow readsorption in line with the kinetics shown in Figure 1B. The intermediate final value of the interfacial tension suggests that some particles remain adsorbed on the surface of the oil droplets at $\mathrm{pH} 3$. In contrast, the colloidosome proved to be immune to the same $\mathrm{pH}$ jump, which indicates effective cross-linking of the interface by the PPG-TDI. Hindered swelling of the cross-linked particles at the interface is expected to occur upon exposure to acid.

Giant colloidosomes consisting of adsorbed PTBAEMA latex particles were formed at $\mathrm{pH} 10$ and aged for $60 \mathrm{~s}$. These conditions are known to produce stable colloidosomes, as previously reported. ${ }^{30}$ The $\mathrm{pH}$ of the aqueous continuous phase was then quickly adjusted to $\mathrm{pH} 3$ and the giant microgel colloidosomes were brought into contact. No coalescence was

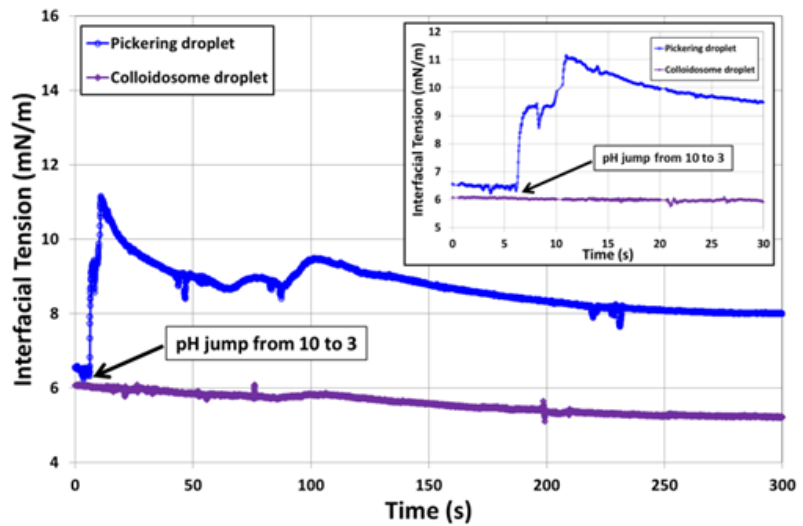

Fig. 6. Dynamic interfacial tension of an isopropyl myristate droplet aged for 360 $\mathrm{s}$ at $\mathrm{pH} 10$ (in the presence of a $3.8 \times 10^{-2} \% \mathrm{w} / \mathrm{v}$ aqueous dispersion of chargestabilised PTBAEMA latex particles with $0.01 \mathrm{M} \mathrm{KNO}_{3}$ background electrolyte) undergoing a $\mathrm{pH}$ jump from 10 to 3 in both the absence $(0)$ and presence $(\diamond)$ of an oil-soluble cross-linker, PPG-TDI.

observed when left in contact for 5 min for either oil, as shown in Figure 7 (ESI $\uparrow$ Figure S6 for sunflower oil). In principle, additional adsorption could occur outside the contact zone during this period. Nevertheless, the giant colloidosomes could be subsequently separated intact and exhibited no sign of crosslinker transfer across the contact zone, as evidenced by the absence of any interconnecting 'neck' or 'bridge'. This is in striking contrast to previous observations reported by Morse and co-workers at $\mathrm{pH} 10 .{ }^{30}$ Thus we hypothesise that the contact zone contains swollen microgel particles, whose high degree of hydration prevents diffusion of the water-insoluble PPG-TDI (see Figure 8). In a further set of experiments, the giant colloidosomes were brought into contact a second time and then

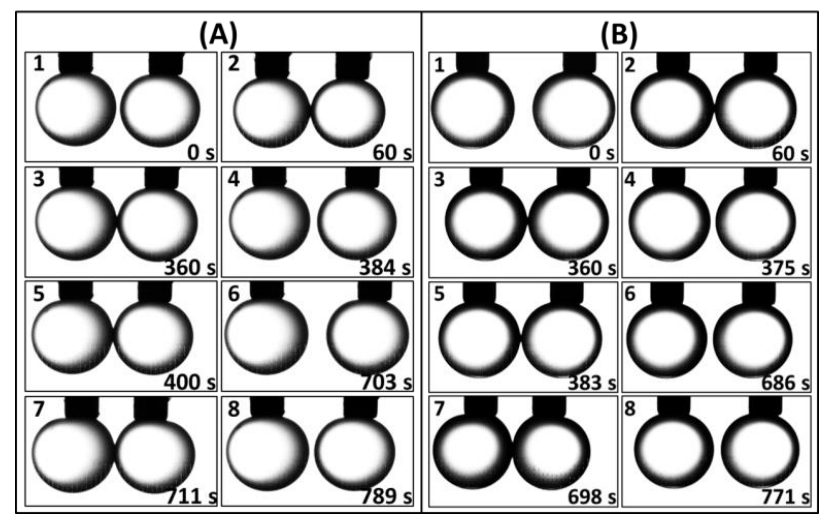

Fig. 7. Sequence of images obtained for two pendant colloidosomes showing contact and separation with no inter-colloidosome cross-linking. The colloidosomes were formed when (A) $n$-dodecane or (B) isopropyl myristate droplets containing PPG-TDI cross-linker were exposed to a $1.6 \times 10^{-2} \mathrm{wt} \%$ aqueous dispersion of charge-stabilised PTBAEMA latex with $0.01 \mathrm{M} \mathrm{KNO}_{3}$ background electrolyte. Droplets were aged in isolation at $\mathrm{pH} 10$ for $60 \mathrm{~s}$ before the $\mathrm{pH}$ was adjusted to 3 (Image 1 ) and the microgel colloidosomes were brought into contact (Image 2). After 5 mins in contact (Image 3) the droplets were moved apart stepwise using a linear actuator with a step size of $0.15 \mathrm{~mm}$ (Image 4). No intercolloidosome cross-linking or coalescence was observed. The microgel colloidosomes were then brought into contact again (Image 5) for a further 5 mins before being separated (Image 6). The colloidosomes were then forced into close proximity (Image 7) for $60 \mathrm{~s}$ more to increase their contact area, before being separated (Image 8). 

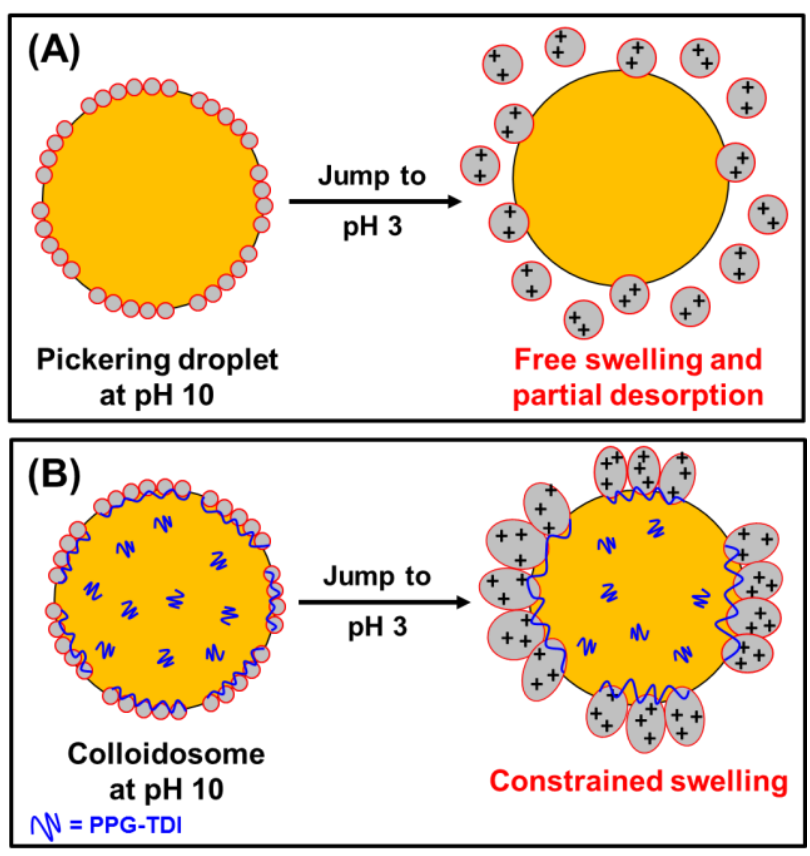

Fig. 8. Schematic representation of the effect a pH jump from 10 to 3 has on the oil/water interface of both (A) a PTBAEMA-stabilised Pickering droplet, and (B) a covalently cross-linked PTBAEMA colloidosome.

a third time, but with greater force and thus contact area (see Figure 7, Image 7), held for $60 \mathrm{~s}$ and then easily separated intact (see Figure 7, Image 8). These observations confirm that negligible cross-linker diffusion occurred under these conditions.

These observations provide a rational basis for the formation of robust colloidosomes. For a highly cross-linked interface comprising a close-packed layer of PTBAEMA latex particles, the colloidosomes should be prepared at $\mathrm{pH} 10$. If this is conducted at a sufficiently high volume fraction, some degree of inter-colloidosome adhesion and possibly fusion will occur. ${ }^{30}$ However, if the $\mathrm{pH}$ of the aqueous continuous phase is lowered to $\mathrm{pH} 3$ after colloidosome formation, the stability of individual colloidosomes can be preserved in their microgel form.

In principle, this route should confer far greater stability owing to the strong electrosteric repulsion between colloidosomes, which appears to retain an aqueous thin film between contacting colloidosomes. If the desired $\mathrm{pH}$ for a given application is alkaline, this could be adjusted immediately prior to use with minimal change in the colloidosome volume fraction. Conversely, stable microgel colloidosomes can be formed at $\mathrm{pH}$ 3 , but the interfacial coating is expected to be far less robust under these conditions. ${ }^{17}$

\section{Conclusions}

The preparation, interaction dynamics and stability of pairs of giant Pickering droplets and colloidosomes stabilised by swollen cross-linkable PTBAEMA microgel particles has been characterised for three different oils: $n$-dodecane, isopropyl myristate and sunflower oil. Adsorption of the swollen microgel particles was confirmed by dynamic interfacial tension and rheological measurements on single pendant oil droplets, and assessment of the oscillatory dynamics for coalescing droplet pairs. Like the analogous bulk emulsions,${ }^{24}$ microgel adsorption does not prevent coalescence of the giant Pickering droplet pairs. This instability can be attributed to the significantly lower curvature of the giant Pickering droplets investigated here. However, the presence of the PPG-TDI cross-linker within the oil phase resulted in the formation of robust colloidosomes. Differences in the stability of the colloidosomes for the three oils were observed as a function of the interface ageing time. Isopropyl myristate-based colloidosomes were the least stable, while $n$-dodecane-based colloidosomes proved to be the most stable. This reflects the balance between the adsorption kinetics of the PPG-TDI cross-linker and the microgel particles, which must both be present at the interface to form a stable colloidosome.

Mutual adsorption was virtually instantaneous in $n$-dodecane but took up to $120 \mathrm{~s}$ in the case of isopropyl myristate. The impact of an acid-induced latex-to-microgel transition on the interaction between giant colloidosomes formed at $\mathrm{pH} 10$ was also studied. The acid challenge did not result in coalescence, which is consistent with previously reported observations for the closelyrelated bulk emulsion. ${ }^{25}$ Unlike colloidosomes formed from the same particles in their non-swollen latex state at $\mathrm{pH} 10$, there was no evidence for inter-colloidosome cross-linking. This suggests the presence of an aqueous film separating the two giant colloidosomes when they are brought into close proximity. Drainage of this thin film is retarded by the presence of swollen, hydrophilic cross-linked microgel particles at the two adjacent interfaces. These microgel colloidosomes are more robust than those formed from swollen particles adsorbed at $\mathrm{pH} 3$ and subsequently cross-linked.

\section{Acknowledgements}

EJW and SA would like to thank the Australian Research Council for DP120102305. We thank the EPSRC for a PhD studentship and Cytec (Drogenbos, Belguim) for CASE support for AJM. METRC is also thanked for partial financial support of AJM's PhD studentship.

\section{Notes and references}

1. O. D. Velev, K. Furusawa and K. Nagayama, Langmuir, 1996, 12, 23742384.

2. O. D. Velev, K. Furusawa and K. Nagayama, Langmuir, 1996, 12, 23852391.

3. H. N. Yow and A. F. Routh, Soft Matter, 2006, 2, 940-949.

4. J. W. Salari, J. van Heck and B. Klumperman, Langmuir, 2010, 26, 14929-14936

5. S. Laib and A. F. Routh, Journal of Colloid and Interface Science, 2008, 317, 121-129.

6. M. F. Hsu, M. G. Nikolaides, A. D. Dinsmore, A. R. Bausch, V. D. Gordon, X. Chen, J. W. Hutchinson and D. A. Weitz, Langmuir, 2005, 21, 2963-2970.

7. A. D. Dinsmore, M. F. Hsu, M. G. Nikolaides, M. Marquez, A. R. Bausch and D. A. Weitz, Science, 2002, 298, 1006-1009.

8. J. Li and H. D. H. Stoever, Langmuir, 2010, 26, 15554-15560.

9. V. D. Gordon, C. Xi, J. W. Hutchinson, A. R. Bausch, M. Marquez and D. A. Weitz, Journal of the American Chemical Society, 2004, 126, 1411714122. 
10. P. F. Noble, O. J. Cayre, R. G. Alargova, O. D. Velev and V. N. Paunov, Journal of the American Chemical Society, 2004, 126, 8092-8093.

11. O. J. Cayre and V. N. Paunov, Journal of Materials Chemistry, 2004, 14, 3300-3302.

12. W. Chen, X. Liu, Y. Liu and H.-I. Kim, Colloid and Polymer Science, 2010, 288, 1393-1399.

13. S. A. F. Bon, S. Cauvin and P. J. Colver, Soft Matter, 2007, 3, 194-199.

14. T. Chen, P. J. Colver and S. A. F. Bon, Advanced Materials, 2007, 19, 2286-2289.

15. H. Skaff, Y. Lin, R. Tangirala, K. Breitenkamp, A. Boker, T. P. Russell and T. Emrick, Advanced Materials, 2005, 17, 2082-2086.

16. Y. Lin, H. Skaff, A. Boker, A. D. Dinsmore, T. Emrick and T. P. Russell, Journal of the American Chemical Society, 2003, 125, 12690-12691.

17. K. L. Thompson, M. Williams and S. P. Armes, Journal of Colloid and Interface Science, 2015, 447, 217-228.

18. L. M. Croll and H. D. H. Stover, Langmuir, 2003, 19, 10077-10080.

19. L. M. Croll and H. D. H. Stover, Langmuir, 2003, 19, 5918-5922.

20. K. L. Thompson and S. P. Armes, Chemical Communications, 2010, 46, 5274-5276.

21. M. Williams, S. P. Armes, P. Verstraete and J. Smets, Langmuir, 2014, 30, 2703-2711.

22. D. B. Lawrence, T. Cai, Z. Hu, M. Marquez and A. D. Dinsmore, Langmuir, 2007, 23, 395-398.

23. R. K. Shah, J. W. Kim and D. A. Weitz, Langmuir, 2010, 26, 1561-1565.

24. A. J. Morse, D. Dupin, K. L. Thompson, S. P. Armes, K. Ouzineb, P. Mills and R. Swart, Langmuir, 2012, 28, 11733-11744.

25. A. J. Morse, J. Madsen, D. J. Growney, S. P. Armes, P. Mills and R. Swart, Langmuir, 2014, 30, 12509-12519.

26. W. K. Wang, A. H. Milani, L. Carney, J. F. Yan, Z. X. Cui, S. Thaiboonrod and B. R. Saunders, Chemical Communications, 2015, 51, 3854-3857.

27. N. P. Ashby, B. P. Binks and V. N. Paunov, Physical Chemistry Chemical Physics, 2004, 6, 4223-4225.

28. S. Ata, Langmuir, 2008, 24, 6085-6091.

29. K. L. Thompson, E. C. Giakoumatos, S. Ata, G. B. Webber, S. P. Armes and E. J. Wanless, Langmuir, 2012, 28, 16501-16511.

30. A. J. Morse, S. Y. Tan, E. C. Giakoumatos, G. B. Webber, S. P. Armes, S. Ata and E. J. Wanless, Soft Matter, 2014, 10, 5669-5681.

31. S. Ata, E. S. Davis, D. Dupin, S. P. Armes and E. J. Wanless, Langmuir, 2010, 26, 7865-7874.

32. P. Creux, J. Lachaise, A. Graciaa, J. K. Beattie and A. M. Djerdjev, The Journal of Physical Chemistry B, 2009, 113, 14146-14150.

33. J. K. Beattie and A. M. Djerdjev, Angewandte Chemie-International Edition, 2004, 43, 3568-3571.

34. K. Roger and B. Cabane, Angewandte Chemie-International Edition, 2012, 51, 5625-5628.

35. S. Ata, Journal of Colloid and Interface Science, 2009, 338, 558-565.

36. G. Bournival, R. J. Pugh and S. Ata, Minerals Engineering, 2012, 25, 4753.

37. L. Hoff, P. C. Sontum and J. M. Hovem, The Journal of the Acoustical Society of America, 2000, 107, 2272-2280.

38. C. C. Church, The Journal of the Acoustical Society of America, 1995, 97, 1510-1521.

39. F. Pinaud, K. Geisel, P. Masse, B. Catargi, L. Isa, W. Richtering, V. Ravaine and V. Schmitt, Soft Matter, 2014, 10, 6963-6974.

40. Z. Li, K. Geisel, W. Richtering and T. Ngai, Soft Matter, 2013, 9, 99399946.

41. Y. Wu, S. Wiese, A. Balaceanu, W. Richtering and A. Pich, Langmuir, 2014, 30, 7660-7669.

42. M. Destribats, V. Lapeyre, M. Wolfs, E. Sellier, F. Leal-Calderon, V. Ravaine and V. Schmitt, Soft Matter, 2011, 7, 7689-7698.

43. W. Richtering, Langmuir, 2012, 28, 17218-17229.

44. V. I. Kovalchuk, G. Loglio, V. B. Fainerman and R. Miller, Journal of Colloid and Interface Science, 2004, 270, 475-482.

45. G. Loglio, P. Pandolfini, R. Miller, A. V. Makievski, F. Ravera, M. Ferrari and L. Liggieri, Novel Methods to Study Interfacial Layers, 2001, 11, 439-483. 